\title{
Clinical Study of Pingyangmycin Combined with Nd:YAG Laser to Treat Venous Malformations in the Oral and Maxillofacial Region
}

\section{Li Y*, Yang J, Duan R, Guan X and Lou H}

Department of Oral and Maxillofacial Surgery, The First People's Hospital of Yunnan, The Affiliated Hospital of Kunming University of Science and Technology, China

*Corresponding author: Yongsheng Li, Department of Oral and Maxillofacial Surgery, The First People's Hospital of Yunnan Province, The Affiliated Hospital of Kunming University of Science and Technology, Kunming 650032, Yunnan, China; Email: liyongshengkw@sohu.com

\section{Research Article}

Volume 6 Issue 1

Received Date: February 04, 2021

Published Date: March 08, 2021

DOI: $10.23880 /$ oajds-16000288

\section{Abstract}

Purpose: To explore the efficacy of pingyangmycin (PYM) combined with Neodymium yttrium aluminum garnet (Nd:YAG) laser to treat venous malformations in the oral and maxillofacial region.

Methods: Eighty-six patients were randomly divided into two groups. Forty-two patients were allocated to the experimental group and were injected with PYM at the lesion site, followed by Nd:YAG laser irradiation two days later. Forty-four patients were allocated to the control group and received only Nd:YAG laser irradiation (15-30W of power). All patients came back for a follow-up examination one month later.

Results: Twenty-eight patients in the experimental group (66.67\%) and 16 patients in the control group (36.31\%) had no lesions after one treatment. Additionally, 10 patients in the experimental group (23.81\%) and nine patients in the control group (20.45\%) showed significant improvement at one month post-treatment. Compared to the control group, the experimental group demonstrated superior outcomes and shorter treatment durations. The between-group difference was significant $(\mathrm{p}<0.05)$.

Conclusion: PYM combined with Nd:YAG laser is an effective treatment of venous malformations in the oral and maxillofacial region, potentially shortening treatment durations.

Keywords: Pingyangmycin; Nd:YAG laser; Combination therapy; Venous malformations

Abbreviations: PYM: Pingyangmycin; FGF: Fibroblast Growth Factor; PDG: Platelet Derived Growth Factor.

\section{Introduction}

Approximately $60 \%$ of hemangiomas and vascular malformations occur in the oral and maxillofacial region, and venous malformations are the most common form of these diseases. Venous malformations usually occur in regions involving facial skin, subcutaneous tissues, and oral mucosa such as the tongue, lips, and mouth. Maxillofacial lesions not only affect appearance and function, but they may also become life-threatening due to bleeding and asphyxia [1]. A variety of combination therapies for treating venous malformations have been tested [2-4]. For example, pingyangmycin (PYM) has been used to treat venous malformations, but it requires a treatment cycle of one month or longer, with several injections during each treatment cycle. Although Neodymium yttrium aluminum garnet (Nd:YAG) lasers can achieve immediate and desirable outcomes in the treatment of oral and maxillofacial venous malformations, their repeated use may cause damage. Consequently, we adopted a combination therapy that uses both pingyangmycin and Nd:YAG lasers to treat these lesions. Combination therapy shortened 
the average therapy duration and decreased the times of required treatments. Between 2010 and 2016, we treated 86 patients with venous malformations using PYM and Nd:YAG laser combination therapy and obtained excellent results, which we report below.

\section{Materials and Methods}

\section{Materials}

The Nd:YAG laser instrument (Model ML-3100) was supplied by Wuhan Miracle Laser Company Limited (Hubei, China). The fiber optic cable diameter of the ML-3100 was $0.5 \mathrm{~mm}$. The laser featured a wave length of $1,064 \mathrm{~nm}$ and an output power, after coupling, of $0-100 \mathrm{~W}$, which could be adjusted continuously. PYM was produced by the Tianjin Pharmacy Factory. Its structure is identical to Blemycin A5.

\section{Methods}

86 patients with oral and maxillofacial venous malformations were enrolled in our study between April 2010 and April 2016. There were 41 male patients and 45 female patients with an average age of 16.5 years. The youngest patient was 1 year old and the oldest patient was 35 years old. Among these patients, the venous malformations were localized to various regions including the lips $(n=30)$, the back surface of the tongue $(n=22)$, forehead $(n=12)$, face $(n=11)$, and buccal mucosa $(n=11)$. Lesion sizes ranged from $1.0 \times 1.0 \times 0.3 \mathrm{~cm} 3$ to $4.5 \times 3.5 \times 0.5 \mathrm{~cm} 3$.

These patients had no other diseases or history of drug allergies, except for local venous malformations. The patients were divided into two groups randomly. Among them, 42 patients were allocated to the experimental group and were injected with PYM at the lesion site, followed by Nd:YAG laser irradiation two days later. 44 patients were allocated to the control group and received only Nd:YAG laser irradiation. Regard to prepare the PYM injection, $8 \mathrm{mg}$ of PYM hydrochloride powder was dissolved in $2 \mathrm{ml}$ of $1 \%$ lidocaine to obtain an injection liquid with a concentration of $4 \mathrm{mg} / \mathrm{ml}$. The patients in the experimental group were injected with 0.3 $\mathrm{mg} / \mathrm{kg}$ PYM (the dose of single injection was no greater than $8 \mathrm{mg}$ ) at the lesion site after routine disinfection. Two days later, the patients underwent infiltration anesthesia using $2 \mathrm{ml}$ of $2 \%$ lidocaine and received Nd:YAG laser irradiation treatment from the edge to the center of the lesion. The laser power was $15-30 \mathrm{~W}$, the diameter of the laser spot was $0.2 \mathrm{~cm}$, and the distance from the bottom of the fiber optic cable to the surface of the lesion was $1 \mathrm{~mm}$. The patients in the control group underwent infiltration anesthesia using $2 \mathrm{ml}$ of $2 \%$ lidocaine and received $\mathrm{Nd}$ :YAG laser irradiation treatment as the same way as that in the experimental group. All patients took oral antibiotics for two days after the operation to prevent infections, and they returned one month later for treatment efficacy evaluation according to pre-defined criteria (Table 1).

\begin{tabular}{|c|c|}
\hline Cured & Complete elimination of lesions \\
\hline Significant efficacy & $\begin{array}{c}\text { Lesions reduced by approximately } \\
75 \%\end{array}$ \\
\hline Effective & $\begin{array}{c}\text { Lesions reduced by approximately } \\
50 \%\end{array}$ \\
\hline Ineffective & Lesions reduced by less than $25 \%$ \\
\hline
\end{tabular}

Table 1: Venous malformation treatment efficacy evaluation.

\section{Results}

All 86 patients completed the treatment successfully. The patients in the experimental group showed mild swelling at the PYM injection site that typically abated two days later. After Nd:YAG laser irradiation, the lesions became white, then withered and in many cases disappeared (Figure 1). Figure 2 depicts change of patients with venous malformations before, and after Nd:YAG treatment.

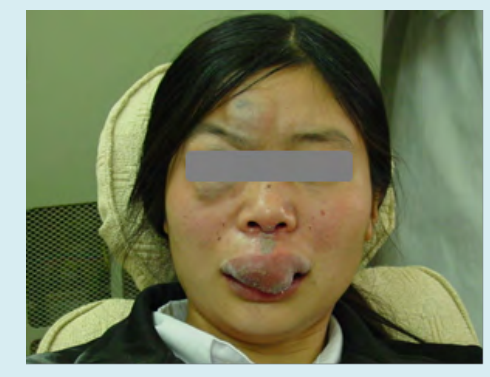

No Treatment

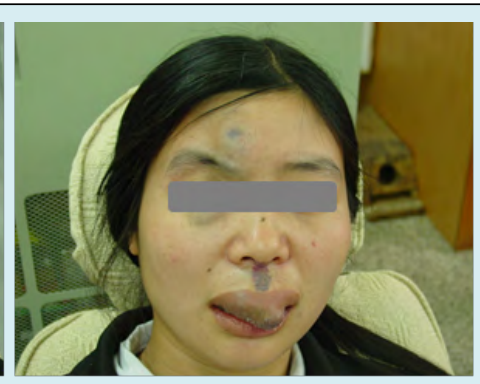

PYM Treatment

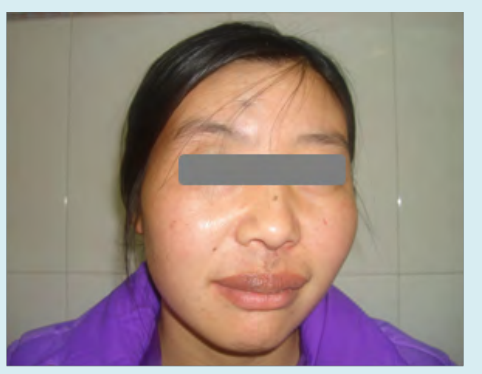

Nd:YAG + PYM Treatment

Figure 1: Pingyangmycin Combined with Nd:YAG laser treatment cured venous malformation. 

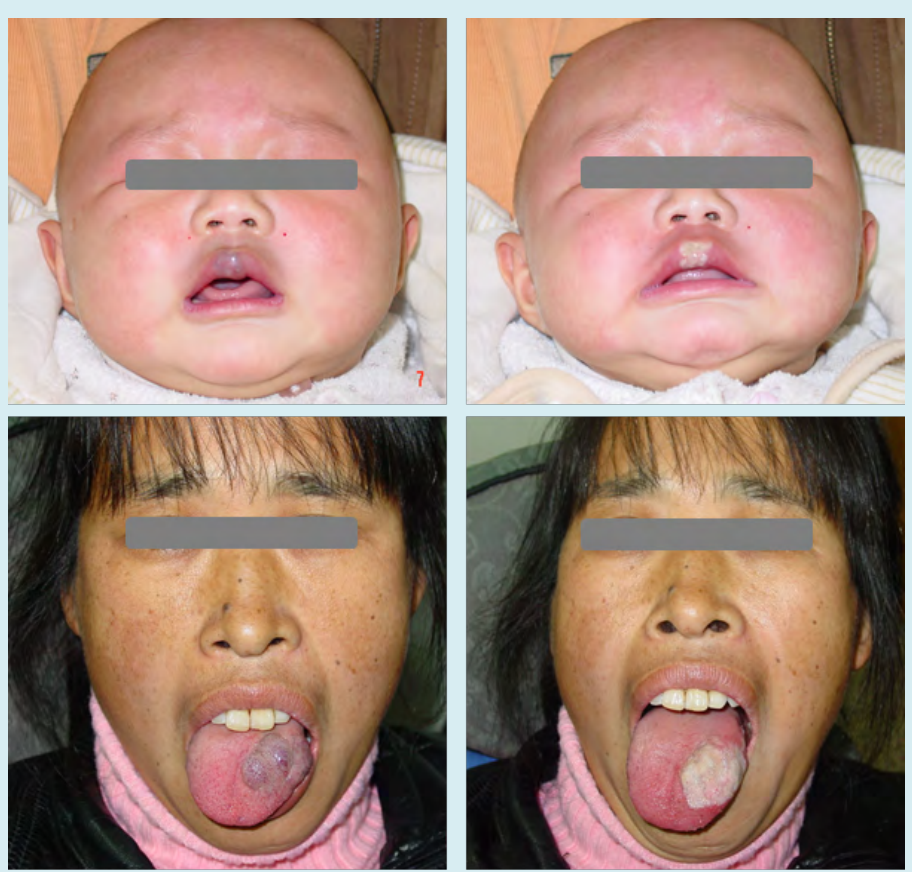

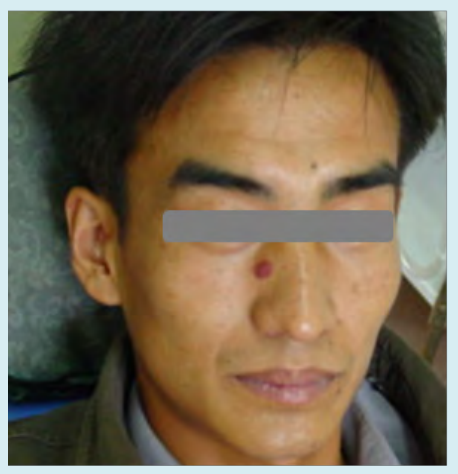

No Treatment

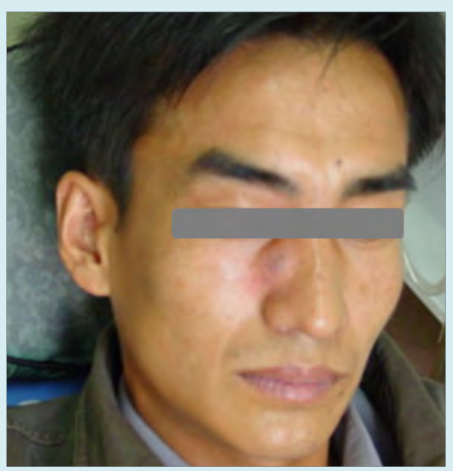

Nd:YAG Treatment

Figure 2: Pre- and post-Nd:YAG laser treatment for patients with venous malformations.

All the patients were observed for one month and we observed significantly different effects at the venous malformation sites in patients who received the combination therapy of PYM injection and Nd:YAG laser irradiation. Ten patients in the experimental group showed mild swelling after PYM injection. However, obvious changes were seen in almost patients after Nd:YAG laser irradiation. In general, blisters started to appear after one hour of irradiation, accompanied by congestion and swelling that peaked after 24 hours. Subsequently, a scab would form on the lesion surface and detach approximately two weeks later. Treatment efficacy results for all 86 patients are shown in Table 2.

\begin{tabular}{|c|c|c|}
\hline & Nd:YAG + PYM (n) & Nd:YAG only (n) \\
\hline Cured & 28 & 16 \\
\hline Significant efficacy & 10 & 9 \\
\hline Effective & 5 & 9 \\
\hline Ineffective & 1 & 7 \\
\hline
\end{tabular}

Table 2: Treatment efficacy in 86 patients with venous malformations. 


\section{Open Access Journal of Dental Sciences}

The experimental group showed a better efficacy and a shorter treatment duration, compared with the control group. There was significant $(\mathrm{p}<0.05)$ difference in the efficacy between the two groups as shown by the $\chi 2$ test.

\section{Discussion}

There are many methods for treating venous malformations. Malformations with a limited range, a shallow location, and a low flow volume are commonly treated with PYM injection or Nd:YAG laser irradiation alone.

PYM is an anti-tumor antibiotic that is structurally like Bleomycin A5. In 1969, PYM was separated and purified from the soil of the Pingyang region of the Zhejiang province in China. It is widely used to treat venous malformations and is considered efficacious. As an antibiotic and cellular toxin, PYM can induce platelet accumulation and thrombus formation by suppressing the proliferation of epithelial cells and injuring the endothelial membrane of blood vessels. At the same time, it can cause epithelial edema and trigger inflammation. This thickens the vascular wall and causes vascular occlusion, eventually leading to the denaturation of hemangioma fibers. PYM treatments can take a long time when used in isolation to treat venous malformations; most patients require several treatments, spanning one or more months, to achieve a good treatment outcome.

The Nd:YAG laser is an infrared ray with a wavelength of $1,064 \mathrm{~nm}$. It can penetrate approximately $4.7 \mathrm{~mm}$ of soft tissue, reaching up to $1 \mathrm{~cm}$ in depth and is therefore an ideal light source for treating venous malformations [5]. The laser can be absorbed by red, blue and black tissues. It can also be selectively absorbed by hemoglobin and will not be absorbed by adjacent normal tissues. When hemangioma is irradiated by a Nd:YAG laser, the lesions are quickly destroyed without damaging the normal tissue. The laser can produce a high temperature and produce tissue dehydration, resulting in blood vessel stenosis, blocked circulation, decreased blood flow rate, and increased blood viscosity. It can also lead to local acidosis and degeneration of blood vessel epithelial cells. When the tissues undergo anoxic necrosis, the lesion will disappear. Blood vessels with a diameter of 2-4 mm can be successfully occluded and the irradiating region will become white, yellow or coarse within several minutes.

Although PYM can be used to treat venous malformations, its underlying mechanism remains unclear. Possible mechanisms of action include:

a) Cytotoxicity: The formation of DNA-Fe-PYM complexes with Fe ions and DNA promotes the oxidation of bivalent $\mathrm{Fe}$ into trivalent $\mathrm{Fe}$, resulting in DNA strand breaks and simultaneously generating oxygen free radicals $\mathrm{COH}-$ ) which will directly bind to DNA and change its base, inhibit DNA synthesis, break the DNA chain, interfere with cell division and proliferation, and induce apoptosis [6].

b) Inhibition of endothelial cell proliferation: Atudies have shown that low concentrations of PYM cannot significantly inhibit the proliferation of endothelial cells in vitro, and high concentrations of PYM significantly inhibited the proliferation of endothelial cells [7]. This may be due to the fact that a high in vitro concentration of PYM in endothelial cells will lead to rapid DNA breakage and affect its repair, resulting in suspended transcription and induction of apoptosis [8], although the potency of PYM will decrease after a certain period of time [9].

c) Vascular endothelial injury: $\mathrm{OH}$ - is the most active and the most powerful oxygen free radical in the body. It can directly damage the cell membrane, interfere with ion transport functions across the cell membrane, and lead to intracellular calcium overload. It can activate sensitive ATP enzymes, so that ATP will be excessively consumed; however, the activation of protease and phospholipase will damage the plasma membrane. At the same time, the calcium overload will accumulate a large amount of mitochondrial $\mathrm{Ca}^{2+}$ to reduce its energy production, thus producing vascular endothelial injury [10]. Under normal circumstances, the integrity of the vascular endothelium surface can prevent platelet adhesion.

\section{This is mainly due to:}

- The vascular endothelial layer surface has a thin and smooth lining of endothelial cells, thus preventing contact between circulating platelets with the vascular endothelium;

- A layer of negatively charged protein molecules is adsorbed on the endothelial surface, which can repel platelets and coagulation factors;

- Vascular endothelial cells can produce PGI2 and inhibit the adhesion of platelets.

However, during endothelium injury, the endothelial cell lining will be destroyed, and the number and distribution of ion charges on the endothelial membrane will change. As the result, the endothelial tissue will almost no longer produce PGI2. Because of this, the platelets flowing through the injury site will be immediately activated by exposed subendothelial tissues such as collagen fibers, and quickly adhere to the exposed sub-endothelial tissues. After the platelets are activated, they will release adenosine diphosphate and other inducers, which will promote platelet aggregation and the formation of thrombosis. Secondly, the exposed collagen fibers can activate XII factor, release tissue coagulation factors, start the endogenous and exogenous coagulation system, and promote blood coagulation to form a thrombus. Vascular endothelial injury can also degrade endothelial cells, causing them to proliferate and producing aseptic 


\section{Open Access Journal of Dental Sciences}

inflammation [11]. Strong fibroblast growth and increased levels of fibroblast growth factor (FGF) and platelet derived growth factor (PDG) promote the synthesis of collagen [12]. Consequently, the fiber tissue of the vascular wall will increase and cause vascular wall become thicker, ultimately leading to tissue fibrosis $[13,14]$.

The mechanism of the Nd:YAG laser can be described via the following way $[15,16]$ :

a) Nd:YAG laser's energy can be absorbed quickly by a large number of accumulated red blood cells and hemoglobin within vascular malformations, producing vasoconstriction, which will further induce ischemia and hypoxia. As the result, the vascular endothelium is damaged or lost. This is typically accompanied by platelet aggregation, thrombosis formation in the lumen, and lumen occlusion. Eventually, vascular malformations will shrink.

b) Lasers with high energy density can exert a strong biological effect on the visible components of the blood (i.e., white blood cells, red blood cells, and platelets) [17].

- This will reduce the charge density on the surface of blood cell membrane, resulting in the aggregation of platelets and erythrocytes, increasing blood viscosity. As the result, blood flow slows, and platelets and thrombin are activated, eventually causing thrombosis.

- Activation of neutrophils will produce hydrogen peroxide and its derivatives, inducing dissolution of the vascular endothelial cells and vascular wall injury, thus increasing platelet adhesion.

- Destruction of the blood cell membrane will cause decreased cell deformability, increased cell rigidity, and brittleness, thus making the shape of the blood cell irregular, and causing short protrusions to form on the surface of blood cells. When the energy reaches a certain level, the cell membrane will rupture and lead to hemolysis, which will in turn trigger the release of adenosine diphosphate to induce platelet aggregation and the formation and progression of thrombosis.

c) The high thermal energy released by the laser can dehydrate a large number of cells in the vascular tissues and vascular wall, thus causing stenosis or occlusion in the blood vessel, blocking circulation, decreasing blood flow rate, and increasing blood viscosity. It can also lead to local acidosis and degeneration of vascular endothelial cells, tissue hypoxia necrosis, and lesion disappearance.

d) Under high heat, the structure of the expanded vascular tissues in superficial venous malformations will be damaged, thus inducing fibrosis of the surrounding tissue and sclerosis of the blood vessel wall, which will eventually form scars and shrink venous malformations [18]. e) In addition to its chromatic property, the Nd:YAG laser is also hydrophilic; that is, the laser energy can also be absorbed by the water content in cells and tissues to generate a thermal effect. When the temperature rises $5-10^{\circ} \mathrm{C}$, it can lead to cell damage, followed by inflammation and tissue repair. When the temperature of the tissue is within $100^{\circ} \mathrm{C}$, the macromolecules will be denatured. These macromolecules may undergo breakage of van der Waals bonds, because the denaturation temperature of most proteins is above $60^{\circ} \mathrm{C}$ and the denaturation temperature of DNA is above $70^{\circ} \mathrm{C}$. Above $100^{\circ} \mathrm{C}$, the temperature of the intracellular water content will exceed the boiling point of water, thus causing water evaporation [19].

Injection of PYM alone has some drawbacks. These injections require long treatment durations of a month or even longer. Therefore, it is associated with greater risk and if the number of injections is excessive, it may cause pulmonary fibrosis. In addition, some patients with true hemangioma are non-responsive to PYM injection. On the other hand, treatment by Nd:YAG laser irradiation alone has its own shortcomings. For example, large hemangioma lesions need to be treated by multiple laser irradiations, which can cause local burns and scars. Therefore, PYM injections combined with Nd: YAG laser irradiation may be able to overcome the above-mentioned shortcomings: by slightly increasing tissue damage while reducing side effects at the same time. Our results indicate that this combination treatment is effective for the treatment of venous malformations. However, from the control group results, Nd:YAG laser irradiation alone was less effective than the combination therapy. This suggests that PYM can play a synergistic role with Nd:YAG laser irradiation for the treatment of venous malformations. We believe that the use of Nd:YAG laser irradiation alone is less efficacious for treatment of venous malformations. On the other hand, when PYM injections are used first, followed by Nd:YAG laser irradiation, the efficacy was significantly increased. This suggests that the combination of these two methods can achieve a satisfactory outcome for the patient.

\section{Conclusion}

In conclusion, PYM combined with Nd:YAG laser irradiation is a superior method for treating venous malformations in the oral and maxillofacial region. This combined treatment can shorten treatment duration and reduce or eliminate the need for repeated treatments. Additionally, it reduces the side effects associated with PYM injections or Nd:YAG laser irradiation alone. Future, well-powered studies are required to further consolidate the efficacy of this novel, combined treatment of venous malformations. 
Grants from: This work was supported by the Ten thousand people plan of Yunnan Province, Special project of famous doctors.YNWR-MY-2019-024

\section{References}

1. Corstiaan C, Breugema, Maruschka P, Merkusb J, Smitt JHS, et al. (2004) Quality of life in patients with vascular malformations of the lower extremity. Br J Plast Surg 57(8): 754-763.

2. Zhao J, Zhao Y, Zhang W (2001) Clinical study of using PSD combination therapy to treat hemangioma. Journal of Comprehensive Stomatology 17(2): 128-129.

3. Xu X, Lin $P$ (2001) Local treatment of oral and maxillofacial cavernous hemangioma using Nd: YAG laser and triamcinolone acetonide (a report of 50 cases). Journal of Fujian Medical University 35: 400-401.

4. Huang M, Chen X (2005) Treatment of tongue venous malformations with a combination therapy of pingyangmycin and Nd: YAG laser irradiation. Journal of Modern Stomatology 19(3): 330.

5. Low DW (2003) Management of Adult Facial Vascular Anomalies. Facial Plast Surg 19(1): 113-130.

6. Tai KW, Chou MY, Hu CC, Yang JJ, Chang YC (2000) Induction of apoptosis in KB cells by Pingyangmycin. Oral Oncol 36(2): 242-247.

7. Si Y, Jia J, Jia Y (2006) Effects of pingyangmycin on the expression of adhesion molecules and the proliferation of endothelial cells derived from human vein malformations. Chinese Journal of Oral and Maxillofacial Surgery 4(3): 220-224.

8. Chen C, Zhang Z (2004) Dose and time effect of pingyangmycin in the inhibition of tongue squamous cell carcinoma and possible anti-cancer mechanism. Chinese Journal of Oral and Maxillofacial Surgery 2(4): 261-265.

9. Azuma A, Takahashi S, Nose M, Araki K, Araki M, et al. (2000) Role of E-selectin in bleomycn induced lung fibrosis in mice. Thorax 55(2): 147-152.
10. Bai N, Chen Y (2006) Application and research of pingyangmycin in the treatment of hemangioma. Chinese Journal of Aesthetic Medicine 15(2): 198-200.

11. Ishii H, Takada K (2002) Bleomycin induces E-selectin expression in culturec umbilical vein endothelial cells by increasing its mRNA levels through activation of NF- $\mathrm{BB} /$ Rel. Toxicol Appl Pharmacol 184(2): 88-97.

12. Yamamoto T, Nishioka K (2005) Cellular and molecular mechanisms of bleomycin-induced murine scleroderma: current update and future perspective. Exp Dermatol 14(2): 81-95.

13. Zhao J, Zhao YF, Chen X, et al. (1997) Comparative study on the effect of pingyangmycin and sodium morrhuate on arteriosclerosis. West China Journal of Stomatology 15: 97.

14. Liang J, Cheng L, Hao X (1997) Experimental study on the effect of pingyangmycin on arteriosclerosis. Journal of Practical Plastic Surgery 8: 215.

15. Kelly KM, Choi B, McFarlane S, Motosue A, Jung B, et al. (2005) Description and analysis of treatments for portwine stain birthmarks. Arch Facial Plast Surg 7(5): 287294.

16. Kelly Km, Kimel S, Smith T, Stacy A, Wilson MJH, et al. (2004) Combined photodynamic and photothermal induced injury enhances damage to in vivo model blood vessels. Lasers Surg Med 34(5): 407-413.

17. Fan Z, Zhao F, Wu M (2000) Effects of intracavitary and external carotid arterial Nd: YAG laser irradiation on the hematological parameters in rabbits. Chinese Journal of Laser Medicine 9(1): 13-17.

18. Fisher DM, Chang CJ, Chua JJ, Chen YR, Achauer BM (2001) Potential complications of intralesional laser photocoagulation for extensive vascular malformations. Ann Plast Surg 47(3): 252-256.

19. Shu B, Hao L (2002) Mechanism and practice of treating scar with laser. Chinese Journal of Clinical Rehabilitation 6(8): 1084-1085. 